\title{
Activity Trackers, Wearables, Noninvasive Technologies for Early Detection, and Management of Cardiometabolic Risks
}

\author{
Akshay R. Tate'; Gundu H. R. Rao ${ }^{2 *}$ \\ ${ }^{1}$ AayuSmart Health Systems, Bengaluru, India \\ ${ }^{2}$ Lillehei Heart Institute, University of Minnesota, Minneapolis, USA
}

\begin{abstract}
According to the World Health Organization (WHO), cardiovascular diseases (CVD) are the number one cause of death globally, taking an estimated 18 million lives each year. Framingham Heart Study Group in Boston developed, the very first 10 -year CVD risk prediction model. The WHO recently derived a 10 -year risk prediction model, for fatal and non-fatal CVD events using individual participant data, from the Emerging Risk Factor Collaboration. This CVD risk model, derivation involved participation of 376,177 individuals from 85 cohorts, and 19,333 incident CVD events. Cardiometabolic diseases in general, and metabolic diseases like hypertension, excess weight, obesity, type-2 diabetes, and vascular diseases collectively, contribute to the overall morbidity and mortality related to CVDs. Of the 18 million CVD-related deaths in 2017, more than three quarters were in low-income and middle-income countries. Risk stratification, management, and prevention models can be very important components of disease prevention and control efforts. In view of this very important role the risk assessment plays, we have reviewed and discussed three specific areas of integration of emerging diagnostic technologies,-population level, individual level, and novel ways of collecting, collating, derivation, and validation of individual risks, risk scores for cluster of risks, and for the use of such data for treatment management. For collection of massive data from general population, we have discussed the National Institutes of Health's flagship program, -All of Us. For empowering individuals in risk assessment and management of identified risks, we have discussed ambulatory diagnostic devices, blood pressure monitors, and continuous glucose monitors. As an example of futuristic approach for the integration of diagnostic tools, we have discussed the products developed by LD-Technologies, Miami, Florida by Dr. Albert Maarek and associates. There are thousands of mHealth apps available for download, and not all are of great use in developing, a seamless diagnostic platform, that we at AayuSmart are trying to put together for cardiometabolic risk stratification, risk prediction, and risk management. We have provided, just a brief glimpse of available tracking, computing, collating, and the software analytical capabilities that exists, and discussed how innovators are trying to use them to build novel diagnostic tools. Despite the important role of telemedicine and internet of things (IOT) in disease management, and healthcare delivery, we have not discussed those applications. In this overview, we have discussed the use of emerging diagnostic tools, for obtaining data at the population level, personal level, as well as for clinical settings. Having said that, we must inform the readers, that there is a great difference between the consumer devices (wrist-worn activity trackers), medical devices (FDA cleared or CE Marked), and a variety of technologies that are in use, but not yet considered clinical testing devices.(International Journal of Biomedicine. 2020;10(3):189-197.)
\end{abstract}

Key Words: cardiovascular diseases • noninvasive technologies $\bullet$ cardiometabolic risk

\section{Introduction}

Cardiometabolic diseases, such as hypertension, obesity, type 2 diabetes (T2D), and vascular diseases have increased in their prevalence and incidence, to epidemic

*Contact Information: Emeritus Professor Gundu H.R. Rao, Laboratory Medicine and Pathology, Director, Thrombosis Research, Lillehei Heart Institute, University of Minnesota. 12500 Park Potomac Ave, Unit 3 06N, Potomac MD 20854.E-mail: gundurao9@gmail.com proportions worldwide. ${ }^{(1-10)}$ Considering the rates of severe obesity in children in the USA, in 2010, the US Whitehouse established the first ever Task Force on childhood obesity, to end this public health problem, within one generation. Followup reports indicate, significant progress in communities across the nation. Obesity is a major risk factor, for the development of T2D. Added to this observation, it is worthwhile considering another contributing factor, pre-diabetes. According to a NHANES 2011-2012 survey, prevalence of prediabetes was $36.5 \%$ among US adults, which is three-fold greater 
than diabetes incidence in the USA. Obesity and diabetes contribute significantly, to the development of vascular pathology. They produce a variety of structural and functional alterations. Like the obesity and diabetes, CVD burden varies among the US states. ${ }^{(10)}$ American Diabetes Association says, over 30 million Americans live with diabetes; 84 million more have prediabetes and the epidemic keeps growing. Unless we act now, one in three Americans will be diagnosed with this disease by 2050. The Sustainable Development Goals also include, a target to reduce the proportion of premature deaths due to NCDs, including diabetes, by one third by 2030. Global Burden of Disease Study 2010 (GBD2010), a collaborative project led by the Institute for Health Metrics and Evaluation (IHME), at the University of Washington, announced its findings to the Royal Society London. Robust management of modifiable risk factors for CVD development, have reduced significantly CVD-related deaths in industrialized nations. Following a healthy diet with increased physical activity, seems to be the most effective, least expensive choice for reducing, or reversing this disease burden.

President Barack Obama announced a research initiative in 2015, that aims to accelerate progress towards a new era of precision medicine (www.whitehouse.gov/precisionmedicine). "Tonight, I'm launching Precision Medicine Initiative to bring us closer to curing diseases like cancer and diabetes- and to give all of us access to the personalized information we need to keep ourselves and our families healthier."(11) Precision medicine is a personalized medicine, that has been further refined by inputs from diverse fields such as genetics, epidemiology, and pharmacogenomics. Part of the effort of this grand visionary initiative was "All of Us, Research Program. ${ }^{(12)}$ The All of Us research program aims, to enroll a diverse group of as least one million persons in the United States, in order to accelerate biomedical research and improve health. The program aims to make the research results accessible, to participants and it is developing new approaches to generate, access, and make data broadly available to approved researchers. Elements of the program protocol include health questionnaire, electronic records (EHRs), physical measurements, the collection and analysis of biospecimens. This one of a kind projects, with over a billion-dollar budget is what we consider a 'Top Down Approach' to the precision medicine, because this program according to the article, does not focus on any set of disease or health status. Having said that, there is a component of the program, which is novel and useful. Participants of this program will have the opportunity, to contribute data from sensors and mobile health devices, and be contacted for future research opportunities. In other words, the All of Us cohort will thereby provide data for prospective, retrospective, and cross-sectional analyses.

Fitbit Inc. has been selected to provide wristband activity trackers, for the National Institutes of Health's largestever research programs, - All of Us, an ambitious effort to recruit and collect data from 1 million people in the USA. The program according to reports, collects genetic information from participants, and uses wearable devices to track healthrelated metrics, including heart rate and sleep patterns. We and others believe that wearables, real-time big data acquisition, software analytics, artificial intelligence, machine learning, and algorithms are going to be a large part of the future digital health. Simple measurements like heart rate and sleep patterns, can indeed provide valuable information about physical activity, performance, and overall wellness. In a press release, (05/13/2017) Dr Conor Heneghan, sleep research scientist at Fitbit, presented his findings of the study, "Estimation of sleep stages using cardiac and accelerometer data from a wrist-worn device at SLEEP-2017, the joint conference of the American Academy of Sleep Medicine and Sleep Research Society in Boston. According to these experts, analysis of 4 billion nights of Fitbit sleep data supports scientific theories, that sleeping $7+$ hours can positively affect sleep quality. Fitbit, the leader in the connected health and fitness wearables, has validated the ability of wrist-worn trackers that incorporate movement and cardiac sensors, like 'Fitbit Alta HR', 'Fitbit Blaze, and Fitbit Charge 2', to accurately determine light, deep, and REM sleep stages.

Fitbit is sold in 110 countries and over 28 million people use it to track their activity. Twenty million people use Fitbit Community FEED app. Individuals who are 18 or older and use smart activity trackers, such as Fitbit, Apple Watch, Amazfit, Garmin Watch, can join the Scripps Translational Science Institute (STSI), study and consent to share their data by down loading the MydataHelps mobile app (www.detectstudy.org). STSI is now partnering with Fitbit to enable users to synch their mHealth wearables, with the national All of Us research program. In addition to this partnership, Fitbit has launched Fitbit Heart Study, its first large-scale virtual study, to validate the use of wearable technology, to identify episodes of irregular heart rhythm suggestive of atrial fibrillation (AF). To track the heart rate, Fitbit's devices use optical heart rate monitoring, or photoplethysmography (PPG) technology, to measure the rate of blood flow directly from user's wrist. Fitbit's algorithm will analyze for irregularities, in the Fitbit Heart Rate Study. The participants who receive a notification about an irregular heart rhythm, will be communicated with a doctor for a virtual appointment at no cost, to get more information and may receive an electrocardiogram (ECG) patch in the mail, at no cost to confirm notification.

A few years ago, we were trying to advocate the use of Fitbit for monitoring sleep patterns, as a part of our cardiometabolic disease prevention protocol, at the prestigious Indian Institute of Science (IISc), Bengaluru, India. The occasion was the 'inauguration ceremony' of a collaborative program between the Stanford (USA) Researchers and the staff of IISc. When we proposed our idea of using the Fitbit for following sleep patterns, the Stanford Researcher expressed his disapproval, -for use of a cheap wrist worn activity tracker in a research program. What then made the Fitbit the choice wearable for use in one of the largest studies put together by the prestigious National Institutes of Health (NHI), USA and the Scripps Translational Science Institute? STSI was founded in 2007 with the specific aim, - to individualize healthcare by leveraging the remarkable progress made in human genomics and combining it with the power of wireless digital technologies.

An analysis published by the Federation of American Societies for Experimental Biology (FASEB) Journal found, 
that Fitbit devices are the most commonly used trackers in biomedical research, including published work (89\%), clinical trials $(83 \%)$, and NIH-funded research $(95 \%)$. To-date, more than 470 published studies-have utilized a Fitbit device, which is more than any other consumer wearable brand, including use of wearables in areas such as diabetes, cardiovascular health, oncology, mental health and post-surgery. "Most of what researchers know, is based on intermittent snapshots of health in an artificial setting or based on personal recall," said Steven Steinhubl, MD, Cardiologist and Director of Digital Medicine at STSI. "Through this research program, we'll have access to comprehensive activity, heart rate, and sleep data, that may help us better understand the relationship between lifestyle behaviors and health outcomes, and what that means for patients on an individualized basis." Canadian researchers have validated the accuracy of Fitbit devices. In a systematic review and narrative synthesis, the authors concluded, "Other than for measures in adults with no limitations in mobility, discretion should be used when considering the use of Fitbit devices as an outcome measurement tool in research or to inform health care decisions."(13)

Cardiometabolic diseases are lifestyle diseases, therefore changes in lifestyle, healthy diet, and exercise, go a long way in preventing the development of risk factors for these chronic diseases.

Harvard Medical School, under the title, "Trusted advice for healthier life" sates, that exercising regularly every day if possible, is the single most important thing you can do for your health. In the short term, exercise helps to control appetite, boost mood, and improve sleep. In the long term, it reduces the risk for heart disease, stroke, dementia, depression, and many cancers. ${ }^{(14-24)}$ Several clinical studies, indeed, have demonstrated the benefit of healthy lifestyle in preventing premature mortality due to these diseases. ${ }^{(25-27)}$ The range of health and fitness trackers and wearable technologies, have multiplied since the time Fitbit launched its activity tracker in 2009, reflecting the widespread interest and desire to be physically active. We and others believe, that the fitness technologies have potential, to significantly impact public health, research and policies. ${ }^{(28,29)}$ We suggest in this overview, some of the novel ways these technologies could be integrated for fine tuning the cardiometabolic risk assessment, risk prediction, and risk management. ${ }^{(30-34)}$

\section{Discussion}

Framingham Heart Study (FHS) is a collaborative project of the National Heart, Lung and Blood Institute (NHLBI) of National Institutes of Health (NIH) and Boston University. ${ }^{(35)}$ According to their web site (https://www.framinghamheartstudy. org), in 1948, Framingham heart study (FHS) scientists and participants embarked on an ambitious project, to identify risk factors for heart disease. Today, the study remains a world-class epicenter for cutting-edge heart, brain, bone and sleep research. The Framingham Risk Score is computed, based on findings of the FHS about various risk factors associated with the heart disease $^{(36)}$ Various professional organizations such as American Heart Association, Mayo Clinic, Cleveland Clinic and many other independent sources, have developed heart disease risk calculators. Framingham Heart Study Group from Boston University developed Cox proportional-hazards regression, to evaluate the risk of developing first CVD event using data from 8,491 Framingham participants. Sex specific multivariable risk functions ("general CVD" algorithms) were derived that incorporated age, total and high-density lipoprotein cholesterol, systolic blood pressure, treatment for hypertension, smoking, and diabetes status. ${ }^{(37)}$ Canadian clinicians have published their perspective on the practical use of the Framingham risk score for primary prevention. ${ }^{(38)}$ They have developed a tool for use with Canadian Cardiovascular Society (CCS) guidelines. It will calculate risk scores using new algorithms. It runs on Firefox, Google Chrome, or Internet Explorer and requires JavaScript. It is available at www.palmedpage.com. The Rasmussen Center at the University of Minnesota has, for the past 18 years, has been performing a noninvasive cardiovascular evaluation in individuals, with no history of cardiovascular disease (CVD). The diagnostic study assesses the severity of functional and structural abnormalities in the small arteries, the large arteries, and left ventricle. Preliminary follow-up data have revealed a striking relationship between the Disease Score, which represents the sum of abnormal tests, and the risk of future morbid events. ${ }^{(39)}$ The ten tests of vascular and cardiac health they assess include; sitting blood pressure, treadmill exercise, small artery elasticity, large artery elasticity, optic fundus photo, carotid intima thickness, microalbuminuria, ECG, Left Ventricular ultrasound, B-type naturitic peptide (BNP). Each of these tests are evaluated as normal (Score 0 ), border line (Score 1), and abnormal (Score 2). An individual with ten abnormal score test will have a score of 20 . The authors have screened over 1000 patients, and arbitrarily and temporarily have identified total scores of 0,1 and 2 as low risk, 3 to 5 as moderate risk and score $>6$ as high risk. ${ }^{(40)}$

\section{Noninvasive Diagnostic Tools for Early Detection of Metabolic Risks}

Common metabolic risks that promote the development of chronic metabolic disease include, oxidative stress, alterations in the circulating vasoactive compounds, altered blood flow, increased blood sugar, elevated blood lipids, altered blood flow, endothelial dysfunction, arterial stiffness, subclinical atherosclerosis, hypertension, excess weight, obesity, prediabetes, diabetes and vascular diseases. Founder, Director, Professor Jay Cohn of Rasmussen Center for Cardiovascular Disease (CVD) Prevention says, that earliest biomarker for CVD is the development of endothelial dysfunction. According to him, "No endothelial dysfunction; no vascular disease." Excess weight and obesity could be computed by measuring body mass index or the Hip/Waist ratio. ${ }^{(8)}$ We have described a variety of noninvasive diagnostic tools, that can be employed for early detection of metabolic risks and management of identified risks. ${ }^{(30-34,40-43)}$

Pulse wave velocity (PWV), is measured by devices, that use either probes or cuffs to noninvasively track the speed of blood flow in meters per second. One measurement is taken at the carotid artery and one at the femoral artery, and the difference between the two-calculated by the device- is 
the current gold standard measure for aortic stiffness. At the University of Minnesota, the Heart Disease Prevention group uses CV-Profiler developed by Hypertension Diagnostics of Minneapolis, Minnesota (www.hypertesnsiondignotics.com). PWV Collaborative Group analyzed the data from 12 studies, in a total of 15, 220 subjects with 1779 combined events. Their conclusion was, that adjusting for conventional risk factors, they showed that the predictive value was strongest in those younger than 50 years of age. With aging comes arterial stiffness, comes blood pressure (BP) elevation, comes pulse pressure increase, which can be measured by Family Practitioners. Availability of cost-effective, reliable smart applications that measure arterial stiffness or endothelial dysfunction means, that primary care clinics will be able to utilize this technology. ${ }^{(44)}$ Kelly and associates, at the University of Minnesota assessed subclinical inflammation, insulin and endothelial dysfunction, before and after exercise in overweight children and adolescents. They found, just eight weeks of aerobic exercise improved fitness, HDL cholesterol, and endothelial dysfunction. ${ }^{(45)}$ Researchers from East China Jiaotong University investigated, the effect of exercise intervention on lipid metabolism, vascular endothelial function, adropin levels, and found similar encouraging results. ${ }^{(46)}$ Yet another, major metabolic risk is the altered blood pressure, which could be monitored easily and accurately by using ambulatory blood pressure monitors.

Abbott Diabetes Care has launched an FDA approved continuous glucose monitor (CGM). We had an opportunity to validate this devise, when it was originally launched in India, for following glucose profile of T2D subjects. The press release at that time claimed, "First-of-its-kind flash glucose monitoring system from Abbott, to revolutionize diabetes management in India." India was the first country, to globally launch this technology. Software presents data in a user friendly, visual charts, enabling productive treatment discussions. The Dexcom G6 (www.dexcom.com/G6.CGM) is the first CGM approved as both stand-alone device and one that can be integrated into automated insulin dosing systems. The composite glucose profile shown in Fig 1, is average values for interstitial glucose, collected and computed every fifteen minutes. In this profile, one can see a low of $117 \mathrm{mg} / \mathrm{dl}$ and a high of $205 \mathrm{mg} / \mathrm{dl}$ with a mean of $157 \mathrm{mg} / \mathrm{dl}$. The disposable sensor collects data for a 14-day period and stores it indefinitely. The data can be downloaded to a computer and further analysis can be performed. In Dexcom G6, data are collected every 5 minutes and therefore, one can get more data points in such profiles. Recent issues of JAMA (June 2020) have reported the results of studies on continuous glucose monitoring in Type-1 patients. $^{(47)}$

Another major development in the noninvasive diagnostic tools for monitoring cardiometabolic risks, is a set of novel devices and integrated analytical systems, put together by Dr Albert Maarek and associates of Miami Florida. Products include, a blood oximeter, blood pressure monitor, and a galvanic skin response monitor, all FDA approved devices. Initial launch included, Sudo Path System (for detection of peripheral neuropathy and microcirculation), TM Oxi System (for detection of diabetic autonomic neuropathy and endothelial function), and ES Complex System (for early detection of diabetes related complications and treatment management). These systems were initially validated at the IPC Heartcare, Mumbai by Dr Pratiksha Gandhi and associates. A typical summary of results is presented in digital format (Fig 2) and in graphic representation in Figure 3.

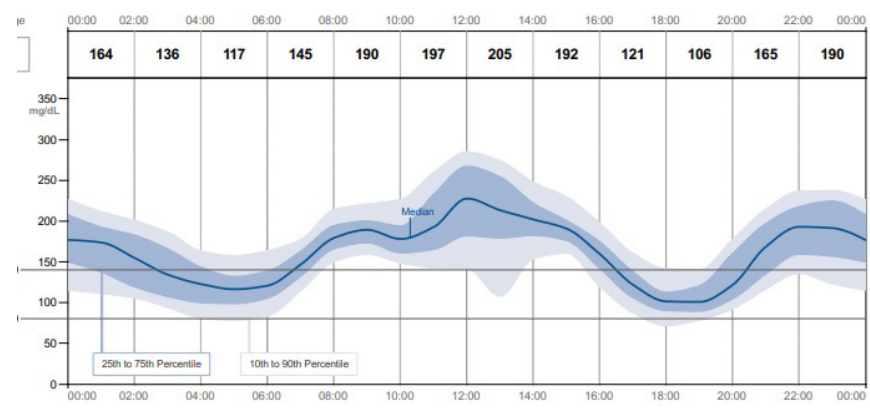

Fig. 1. Interstitial Glucose Profile of an 80-year-old Patient (Personal Data).

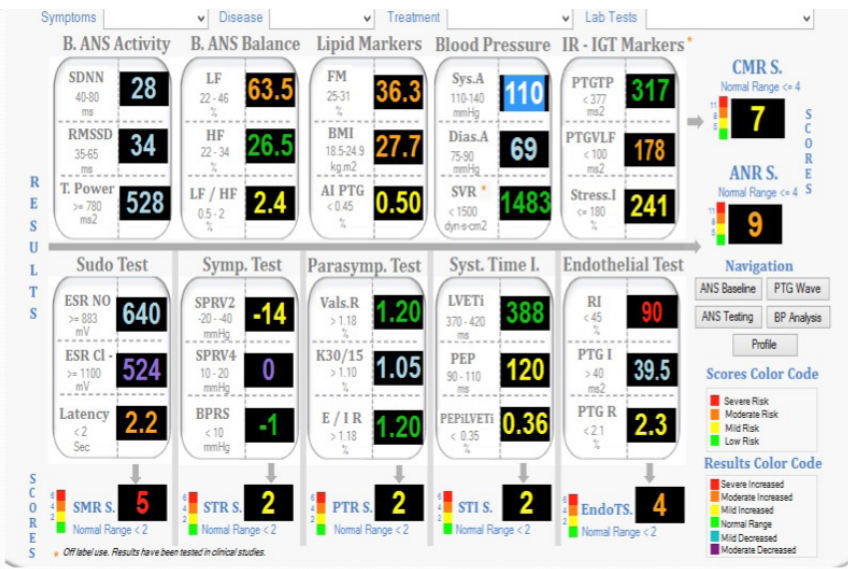

Fig. 2. Summary Results of Cardiometabolic Risk Assessment (Courtesy: Dr Albert Maarek).

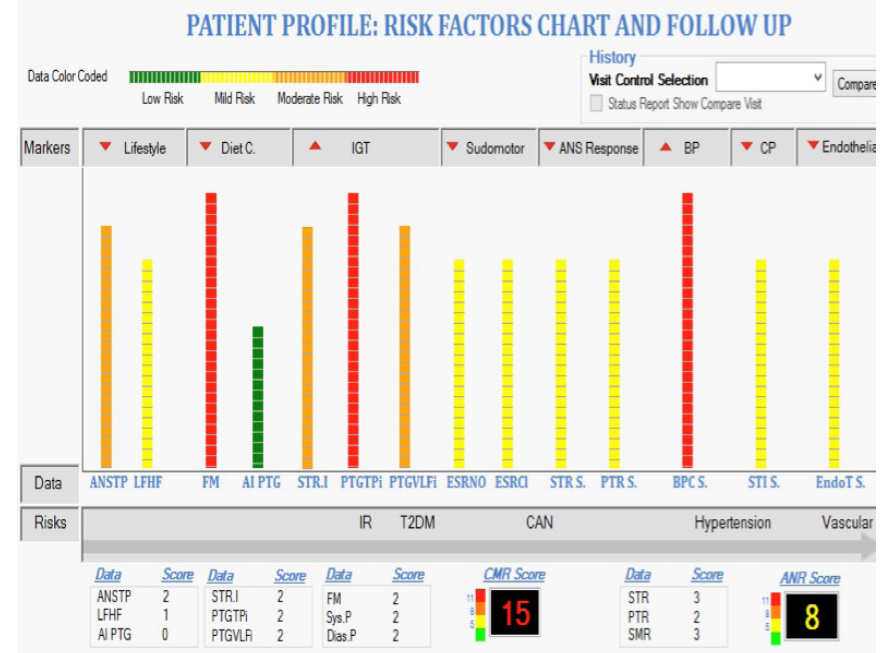

Fig. 3. Summary Results of Cardiometabolic Risks (Courtesy: Dr. Albert Maarek)

Mainly, Sudomotor function evaluation is performed, to detect skin blood flow (microcirculation) and C-fiber density, for early detection of peripheral distal neuropathy in population at high risk. In brief, $\mathrm{C}$-fiber damage could be 
associated with numbness and pain in hands and feet. The spectral analysis, of the photoplethysmography method is non-invasive, fast, operator-independent, and cost-effective, as only an oximeter and galvanic skin response devices are required in order to assess in a single testing, the autonomic nervous system and endothelial function. The spectral analysis techniques used on the photoplethysmogram, as outlined in this study, could be useful when used alongside conventional known cardiovascular disease risk markers. ${ }^{(41)}$

Risk scores computed for various functions tested are digitized and color-coded. Abbreviations used in the following summaries of results include: ANS; Autonomic nervous system, BMI: Body mass index, CAN: Cardiovascular autonomic neuropathy, DAN: Diabetic autonomic neuropathy, CMRS: Cardiometabolic risk score, CVD: Cardiovascular disease, DVP: Digital volume pulse, ESRNO: Electric skin response to nitric oxide, FFT: Fast Fourier transform, FFTPTG: Frequency spectrum of PTG data, FGP; Fasting plasma glucose, GHB: Glycosylated hemoglobin, HRV: Heart rate variability, PNS: parasympathetic nervous system, PTG: Photoplethysmograph, PTGHF: Component associated with R-R changes during various phases of breathing, SNS; Sympathetic nervous system. For additional information about the use of these devices for monitoring cardiometabolic risks, readers are urged to refer to our earlier publications. . $^{(41-43)}$

The bar graph above represents values for the fourteen risk clusters monitored. Cardiometabolic risk score is computed from the values derived by fat mass, spectral analysis of the pulse wave and the blood pressure (Systolic) data. The cardiometabolic risk (CMR Score) is quite high (CMR Score;15) hence shown in red. Individual risk factors that contribute to the overall increase in the CMR also are shown in the bar graph as red.

First risk bar (on the right) reflects the maker before treatment, the second risk bar (on the left) reflects the marker after the treatment (change in lifestyle, diet metformin and antihypertensive medications). In Fig. 4 a summary of results of all the test of a patient are presented in graphic form (CMR Risk Score 15). In the Fig. 4 the data of an early onset diabetic, before and after treatment is presented, showing the ability of the software to compute, compare values before and after treatment and generate composite risk scores (CMR Risk Score 4).

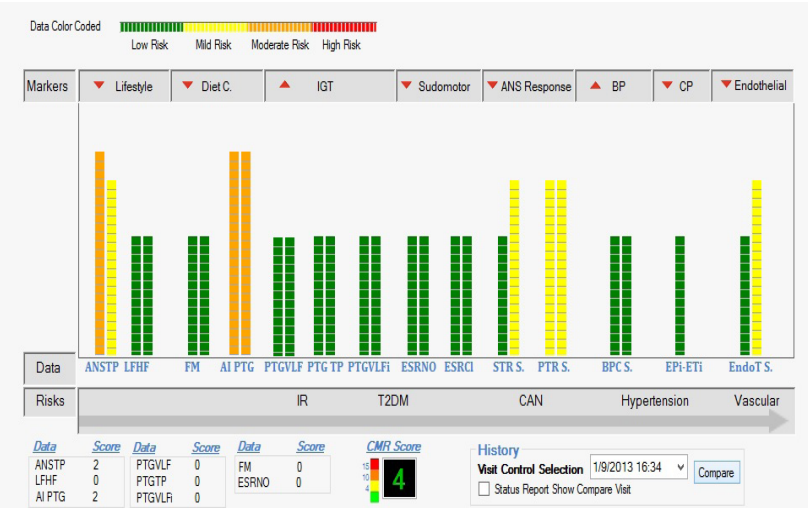

Fig. 4. Patient Profile: Interaction of Markers and Treatment Management. (Courtesy: Dr A, Maarek, LD Technologies, Miami, Florida).

\section{Opportunities, Challenges and Limitations}

American College of Cardiology (ACC) presents a Road Map for innovation on healthcare transformation in the era of digital health, big data, and precision health, presents a shared vision in their Health Policy Statements. ${ }^{(48)}$ In the newly evolving, -novel, patient centered, evidence-driven models, in which rapidly evolving technology-based innovations play a very important role. "These include: digital health with wearable, smartphone, and sensor-based technologies; big data that comprises the aggregation of large quantities of structured and unstructured health information and sophisticated analyses with artificial intelligence, machine learning, and natural language processing techniques; and precision-health approaches to, identify individual-level risk and the determinants of wellness and pathogenicity. "Although there is promise in the development of such innovations, to shift traditional healthcare delivery to virtual and real-time methods and to empower the healthcare enterprise to utilize new technologies and data analytics, there remains a lack of true evaluation of whether these innovations improve outcomes and the quality of care." ${ }^{\prime(49)}$ Looks like there is no way to avoid incorporation of new and emerging technologies, to improve the way the healthcare is delivered in future. Having said that, it is very important discuss the disconnect that exists, between the various stakeholders in this complex healthcare service.

Fifty million people in the USA wear connected devices, to track activity and the number is expected increase to more than 160 million, with the introduction of smart watches. ${ }^{(49,50)}$ There is ever increasing number of direct-to-consumer smart medical devices available to track activity, performance, health status and wellness. We and others have described the use of Photoplethysmographic (PPG) sensors, to estimate the heart rate based on the transmission and absorption of light applied to the skin. Altered flow dynamics and blood volume caused by peripheral pulses, can be measured by dedicated photodetectors and light emitting diodes incorporated in wrist-worn bands or smartphone cameras. Collating data from PPG sensors with accelerometers can facilitate the detection of arrhythmias. ${ }^{(50)}$ Having said that, it is essential to note that activity trackers, wearables although produce massive data on a real- time use, do not provide the needed analytics for computing the performance or risk stratification. According to the experts, even though PPG sensor can detect tachycardia or irregular rhythms, they cannot distinguish between the atrial fibrillation and premature beats, unless the software analytics includes dedicated algorithms that analyze beat-to beat variability. No data analytics and interpretation will ever replace a clinician, in the final decision making. In view of this fact, it will be extremely useful, if there is a collaboration between the innovator and clinicians (end users).

Whereas the ambulatory blood pressure monitors, interstitial glucose monitors, which are US/FDA approved can indeed help the individuals as well as clinicians in monitoring the progression as well as treatment associated regression of these modifiable risks. Continuous glucose monitor is easy to use and relatively affordable and provide real-time data on interstitial glucose profile. The data can be shared by multiple 
users (patients, spouses, clinicians). The use of this device to a great extent empowers the user (diabetic patients) to track the glucose levels and observe in real-time, the effect of lifestyle changes, physical activity, diet, and medications. These types of devices can be used as Theranostic devices, -meaning use for both diagnostics and treatment management purposes. On the other hand, LD-technology products (www.ldteck. com) like SudoPath and TM-Oxi systems provide risk score for over ten different cardiometabolic risks. Except for blood pressure, all other metabolic risks are proprietary softwarederived, therefore warrant clinical studies for validating specificity and efficiency. Having said that, we and others have demonstrated the usefulness of these devices for lifestyle and treatment management. ${ }^{(41-43,51,52)}$ If clinically validated, these noninvasive diagnostic platforms, can be used for diagnosis of early metabolic risks and treatment management of these modifiable risks.

In 2015 according to reliable sources, the market for wearables doubled to reach 1.5 billion USD. This does not seem to be the end of its growth. The market size for these health and activity trackers, is predicted increase to over 50 billion in the next few years. New and better devices are coming all the time. There are great improvements in the sensors and chips used, as well as software analytics and algorithms. Newer wrist-worn trackers (simlemia.com), claim to have incorporated more accurate PPG sensors (3D acceleration sensor) and ECG chips (Nordic 52832) to improve accuracy. They report heart rate, blood pressure, blood oxygen, EKG tracings, sleep monitoring, as well as EKG reports. There are reports, that simple PPG-based screening of ECG by Kardia Mobile (AliveCor) device has been shown, to detect atrial rate and rhythm, AV block, and QRS delay with relative accuracy. Studies have shown that for AF screening, Kardia device has reported sensitivity of $98 \%$ and specificity of $97 \%$ and overall accuracy of $97 \%$ with an optimized algorithm. In our earlier studies, we too have reported excellent sensitivity and specificity of LD-technology risk management systems. ${ }^{(41-44)}$ Murakami and associates from Japan studied, more than dozen wearables including Fitbit and concluded, "Although wearable devices for estimating energy expenditure are becoming increasingly popular, there is little evidence regarding their validity. ${ }^{(53)} \mathrm{A}$ report by experts in the NEJM, discusses the public health benefits and risks of mHealth and the challenges facing the FDA in regulating this technology. ${ }^{(54)}$

\section{Future of Activity trackers, wearables and mHealth}

We at the AayuSmart, India, are quite excited about the opportunities that exists, for the use of emerging diagnostic technologies, for providing better healthcare. ${ }^{(30-34,55-58)}$ The fact that the premier health research institute of the USA, National Institutes of Health (NIH) has partnered with Fitbit for the first digital health technology initiative, to understand the relationships between physical activity, heart rate, sleep and health outcomes, speaks a volume about the opportunities. Furthermore, the launching of bring-your-owndevice (BYOD)program for the NIH's flagship precision medicine program. -All of Us research project, has opened new and novel avenues for big data acquisition, analytics, risk stratification and management. NIH claims that the millionperson precision medicine study 'a constant learning process.' John Reites, chief product officer of Fitbit writes, "We are not only seeing this approach in post-approval research and registries but also phase 11/111 studies." Scripps Translational Science Institute (STSI), has published their first report on, -Inter-and intraindividual variability in daily resting heart rate and its association with age, sex, sleep, BMI, and time of the year: Retrospective, longitudinal cohort study of 92,457 adults.

Cardiogram and Fitbit Inc. have partnered to bring Cardiogram's suite of health monitoring features to Fitbit users around the world, with a goal to improve healthcare management, empower users, and potentially save lives. The Cardiogram app is a digital health and fitness diary, using heart rate and sleep data from wearables and displaying on a user's phone with insights and tracking. Their press release (06/26/2019), claims, "Now, Fitbit users can access Cardiogram's screening tools, which have been clinically validated to detect signs of conditions like diabetes, hypertension, sleep apnea, and atrial fibrillation data from Fitbit services (https://cardiogr.am/research). Fitbit users have the option to upgrade to Cardiogram Premium, - a subscription service that enables sharing live Cardiogram data, insights, and confirmatory tests with a loved one or caretaker, and easily export data to share with a physician. This is just the beginning of digital health application for the improvement of personal healthcare. Cardiogram has validated accuracy of an artificial intelligence-based algorithm (with over 14,000 participants), Deep Heart, to accurately detect multiple health conditions. Steven Steinhubl, the leader of the STSI's All of Us research program concludes that, "Multi-omics longitudinal profiling of individuals, can detect subtle changes in health status at the earliest possible time point, allowing preemptive initiation of mechanism-appropriate, disease-prevention strategies. ${ }^{(57)}$

We at AayuSmart, India, would like to develop a similar personal healthcare platform for the early detection of cardiometabolic risks and management of modifiable risks. We would like to sponsor this project, through our professional society (SASAT) platform (www.sasat.rog). The approach will be like 'All of Us' research project. Any adult participant can register via our App and provide data for further analysis on our personal health portal. Once the data is acquired by any activity tracker, wearable or non-invasive diagnostic tools, our proprietary software with dedicated algorithms will analyze the data and generate risk score for cardiometabolic risks such as excess weight, hypertension, arterial stiffness, endothelial dysfunction, obesity, T2D and vascular diseases. To start with we will use the data from clinically validated diagnostic devices. We will also validate noninvasive diagnostic tools, for the specificity and accuracy in India for standardization purpose. Scripps researchers have launched a new study (DETECT study) to see if data collected from wearable devices, could be used to track flu-like illness, including Covid-19. We have initiated a similar tracking system in India. In addition to tracking severe respiratory 
diseases, we are also tracking prevalence and incidence of cardiometabolic diseases. In a short overview like this, it is rather difficult to discuss all aspects of devices that are useful for future healthcare, readers are urged to refer to reviews and original articles on this topic. ${ }^{(55-63)}$

\section{Conclusions}

There are 325,000 health-related apps available for download from the app store. But only a tiny fraction of those are appropriate for clinical use. This has led the researchers, clinicians, as well as regulatory agencies, to appeal for a better oversight and regulation of the information in these apps. We and others feel, that there is a great disconnect between the entrepreneurs, researchers, software developers, and the end users such as clinicians and the patients. Many apps, for instance, the Fitbit like activity trackers are for that matter, even LD-technology products that we have described, are not seen as high-quality devices for diagnostics by the clinicians. Hardware used for developing this system are oximeter, blood pressure monitors, and galvanic skin response monitor. When the test starts recording the inputs from these devices, software collects the signals from these three devices and saves the recordings for further interpretation. Since this is not a dedicated device, like the ambulatory devices that we have discussed, one wonders how these biomarkers are derived? This is one of the reasons why, we feel that it is very essential for the device developers to work with the clinicians, during the development phase, and incorporate tests that are familiar to the clinicians. Majority of the clinicians use fasting blood glucose (FBG), impaired glucose tolerance (IGT) or HbA1c as gold standards for diabetes management. When they do not see such biomarkers, they feel uncomfortable to use these modern technologies, in their day-to-day disease management. One way to improve this situation is, to develop robust clinical validation for each of the biomarkers computed by software analytics.

Since our interest is in the early detection of risk factors for cardiometabolic diseases, management of modifiable risk factors, we have envisioned use of emerging technologies, at three different phases of prevention protocol. The first phase is population-based studies. We feel the novel approach used in the 'All of Us' program could be used as model. Activity trackers are used by millions of individuals globally and would serve as a useful tool for obtaining massive amounts of data in real-time, on vital functions during various phases of activity. There is a great need to develop appropriate app to collect, collate, and analyze such data to derive useful information on prevalence and incidence of metabolic diseases at the population level. In addition, as is done with Cardiogram app, one can develop appropriate health portals, which can serve as a platform between individuals, care givers, and clinicians. Second level we have discussed in this article is the use of dedicated medical devices, such as ambulatory blood pressure monitors and continuous glucose monitors to empower the patients. The data collected from such devices also could be channeled to the health app for further processing and interpretations. The LD-technology products are novel, noninvasive and easy to use. They have used a sophisticated proprietary software, to compute individual risk factors and further derive cluster of risks and risk scores for cardiometabolic risks (CMR) and autonomous neuro system (ANS) risks. Clinical validation and ability to incorporate such calculated risk scores to fine tune the cardiometabolic risk would be very useful.

There is great interest and excitement in the use of noninvasive sensors, for obtaining data on physical activity, health status, fitness, and wellness. The incorporation of multiple sensors including, accelerometers, gyroscopes, goniometers, oscilloscopes, force sensors (pressure sensors), PPG sensors, can provide a wealth of information as seen in the few examples we have discussed in this overview. What we have outlined in this essay is a novel approach, for integration of emerging technologies, for the development of an affordable healthcare. One area of great importance, if perfected with appropriate clinical validation is, the integration of diagnostic and therapeutic systems into theranostic devices. Majority of devices these days have connectivity to smart platforms. As we have proposed in this article, one can build a health portal, which can collect the needed information on risk factors and available therapeutic interventions. Improved data collection, software analytics can improve the way we diagnose, alert the developing risks, manage and treat metabolic diseases. Thinking about the future of integrated diagnostics, one can visualize a novel precision medicine approach, characterized by the development of sophisticated software programs that will enable to collect, consolidate and integrate a large volume of different sets of data, within the same information system (cardiometabolic diseases), producing a fully integrated report, that will combine genetic, radiology, laboratory and pathology data for developing improved risk stratification and risk management.

\section{Competing Interests} interests.

\section{References}

1. NCD Risk Factor Collaboration (NCD-RisC): Worldwide trends in blood pressure from 1975 to 2015: a pooled analysis of 1479 population-based measurement studies with 19.1 million participants. Lancet. 2017;389(10064):37-55 . doi:10.1016/S0140-6736(16)31919-5

2. Global Burden of Metabolic Risk Factors for Chronic Disease Collaboration: Cardiovascular disease, chronic kidney disease, and diabetes mortality burden of cardiometabolic risk factors from 1980 to 2010: a comparative risk assessment. Lancet Diabetes Endocrinol. 2014;2(8):634-647. doi:10.1016/ S2213-8587(14)70102-0

3. NCD Risk FactorCollaboration: Worldwide trends in diabetes since 1980: a pooled analysis of 751 population-based studies with 4.4 million participants [published correction appears in Lancet. 2017 Feb 4;389(10068):e2]. Lancet. 2016;387(10027):15131530. doi:10.1016/S0140-6736(16)00618-8

4. Kearney PM, Whelton M, Reynolds K, Muntner P, Whelton PK, He J. Global burden of hypertension: analysis 
of worldwide data. Lancet. 2005;365(9455):217-223. doi:10.1016/S0140-6736(05)17741-1

5. Evans A, Tolonen H, Hense HW et al. Trends in coronary artery disease risk factors in the WHO MONICA project. Int J Epidemiol. 2001;30 Suppl 1:S35-S40. doi:10.1093/ije/30. suppl_1.s35

6. Juonala M, Viikari JS, Hutri-Kähönen $\mathrm{N}$, et al. The 21-year follow-up of the Cardiovascular Risk in Young Finns Study: risk factor levels, secular trends and east-west difference. J Intern Med. 2004;255(4):457-468. doi:10.1111/ j.1365-2796.2004.01308.x

7. Reilly JJ, El-Hamdouchi A, Diouf A, Monyeki A, Somda SA. Determining the worldwide prevalence of obesity. Lancet. 2018;391(10132):1773-1774. doi:10.1016/ S0140-6736(18)30794-3

8. NCD Risk Factor Collaboration (NCD-RisC). Worldwide trends in body-mass index, underweight, overweight, and obesity from 1975 to 2016 : a pooled analysis of 2416 populationbased measurement studies in 128.9 million children, adolescents, and adults. Lancet. 2017;390(10113):2627-2642. doi:10.1016/S0140-6736(17)32129-3

9. NCD Risk Factor Collaboration (NCD-RisC). Trends in adult body-mass index in 200 countries from 1975 to 2014: a pooled analysis of 1698 population-based measurement studies with $19 \cdot 2$ million participants [published correction appears in Lancet. 2016 May 14;387(10032):1998]. Lancet. 2016;387(10026):13771396. doi:10.1016/S0140-6736(16)30054-X

10. Global Burden of Metabolic Risk Factors for Chronic Diseases Collaboration (BMI Mediated Effects), Lu Y, Hajifathalian K, et al. Metabolic mediators of the effects of body-mass index, overweight, and obesity on coronary heart disease and stroke: a pooled analysis of 97 prospective cohorts with 1.8 million participants. Lancet. 2014;383(9921):970983. doi:10.1016/S0140-6736(13)61836-X

11. Collins FS, Varmus H. A new initiative on precision medicine. N Engl J Med. 2015;372(9):793-795. doi:10.1056/ NEJMp1500523

12. All of Us Research Program Investigators, Denny JC, Rutter JL, et al. The "All of Us" Research Program. N Engl J Med. 2019;381(7):668-676. doi:10.1056/NEJMsr1809937

13. Feehan LM, Geldman J, Sayre EC, et al. Accuracy of Fitbit Devices: Systematic Review and Narrative Syntheses of Quantitative Data. JMIR Mhealth Uhealth. 2018;6(8):e10527. Published 2018 Aug 9. doi:10.2196/10527

14. Saunders TJ, Atkinson HF, Burr J, MacEwen B, Skeaff CM, Peddie MC. The Acute Metabolic and Vascular Impact of Interrupting Prolonged Sitting: A Systematic Review and Meta-Analysis. Sports Med. 2018;48(10):2347-2366. doi:10.1007/s40279-018-0963-8

15. Biswas A, Oh PI, Faulkner GE, et al. Sedentary time and its association with risk for disease incidence, mortality, and hospitalization in adults: a systematic review and metaanalysis [published correction appears in Ann Intern Med. 2015 Sep 1;163(5):400]. Ann Intern Med. 2015;162(2):123132. doi:10.7326/M14-1651

16. Lynch BM, Owen N. Too much sitting and chronic disease risk: steps to move the science forward. Ann Intern Med. 2015;162(2):146-147. doi:10.7326/M14-2552

17. Berlin JA, Colditz GA. A meta-analysis of physical activity in the prevention of coronary heart disease. Am J Epidemiol. 1990;132(4):612-628. doi:10.1093/oxfordjournals.aje.a115704 18. Eaton CB. Relation of physical activity and cardiovascular fitness to coronary heart disease, Part I: A meta-analysis of the independent relation of physical activity and coronary heart disease. J Am Board Fam Pract. 1992;5(1):31-42.

19. Manson JE, Greenland P, LaCroix AZ, et al. Walking compared with vigorous exercise for the prevention of cardiovascular events in women. $N$ Engl J Med. 2002;347(10):716-725. doi:10.1056/NEJMoa021067

20. Tanasescu M, Leitzmann MF, Rimm EB, Willett WC, Stampfer MJ, Hu FB. Exercise type and intensity in relation to coronary heart disease in men. JAMA. 2002;288(16):19942000. doi:10.1001/jama.288.16.1994

21. Wannamethee SG, Shaper AG, Alberti KG. Physical activity, metabolic factors, and the incidence of coronary heart disease and type 2 diabetes. Arch Intern Med. 2000;160(14):2108-2116. doi:10.1001/archinte.160.14.2108 22. Swain DP, Franklin BA. Comparison of cardioprotective benefits of vigorous versus moderate intensity aerobic exercise. Am J Cardiol. 2006;97(1):141-147. doi:10.1016/j. amjcard.2005.07.130

23. Yu S, Yarnell JW, Sweetnam PM, Murray L; Caerphilly study. What level of physical activity protects against premature cardiovascular death? The Caerphilly study. Heart. 2003;89(5):502-506. doi:10.1136/heart.89.5.502

24. Bove AA. Exercise and Heart Disease. Methodist Debakey Cardiovasc J. 2016;12(2):74-75. doi:10.14797/mdcj-12-2-74

25. Di Cesare M, Bennett JE, Best N, Stevens GA, Danaei G, Ezzati M. The contributions of risk factor trends to cardiometabolic mortality decline in 26 industrialized countries. Int J Epidemiol. 2013;42(3):838-848. doi:10.1093/ ije/dyt063

26. Yusuf S, Hawken S, Ounpuu S, et al. Effect of potentially modifiable risk factors associated with myocardial infarction in 52 countries (the INTERHEART study): case-control study. Lancet. 2004;364(9438):937-952. doi:10.1016/S0140-6736(04)17018-9

27. Khera AV, Emdin CA, Drake I, et al. Genetic Risk, Adherence to a Healthy Lifestyle, and Coronary Disease. N Engl J Med. 2016;375(24):2349-2358. doi:10.1056/NEJMoa1605086

28. Rao GHR. Fitness, Lifestyle changes, Wellness: Cardiometabolic Health. J Cardiol (OAJC). 2018;2(4):000132. 29. Sullivan AN, Lachman ME. Behavior Change with Fitness Technology in Sedentary Adults: A Review of the Evidence for Increasing Physical Activity. Front Public Health. 2017;4:289. Published 2017 Jan 11. doi:10.3389/fpubh.2016.00289

30. Rao GHR. Integration of emerging technologies: Management of cardiometabolic diseases; Review. Ind J Cardio Biol \& Clin Sci. 2018;5(1):111.

31. Rao GHR. Risk prediction, assessment, and management of type-2 diabetes. Point of View. EC Endocrinol Metab Res. 2018;3:30-41.

32. Rao GHR. Integration of novel emerging technologies for the management of type-2 diabetes. Arch Dia. \& Obesity. 2018; 1(1). MIS ID: 000102.

33. Rao GHR. Predictive and Preventive Healthcare: Integration of emerging technologies. J Clin Res In Diab \& Endocrinol. 2018;1(1):1-8.

34. Malmefeldt E, Rao GHR. Noninvasive Diagnostic Tools: Cardiometabolic Risk Assessment and Prediction. J Clin Cardiol Diag. 2019;2(1):1-10.

35. Framingham Heart Study: Three Generation of Dedication. Available at https://framinghamheartstudy.org/

36. Brindle P, Emberson J, Lampe F, et al. Predictive accuracy of the Framingham coronary risk score in British men: prospective cohort study. BMJ. 2003;327(7426):1267. doi:10.1136/bmj.327.7426.1267 
37. D'Agostino RB Sr, Vasan RS, Pencina MJ, et al. General cardiovascular risk profile for use in primary care: the Framingham Heart Study. Circulation. 2008;117(6):743-753. doi:10.1161/CIRCULATIONAHA.107.699579

38. Bosomworth NJ. Practical use of the Framingham risk score in primary prevention: Canadian perspective. Can Fam Physician. 2011;57(4):417-423.

39. Cohn JN. Cardiovascular Disease Progression: A Target for Therapy?. Am J Med. 2018;131(10):1170-1173. doi:10.1016/j.amjmed.2018.03.032

40. Cohn JN, Duprez DA, Grandits GA. Arterial elasticity as part of a comprehensive assessment of cardiovascular risk and drug treatment. Hypertension. 2005;46(1):217-220. doi:10.1161/01.HYP.0000165686.50890.c3

41. Gandhi PG, Rao GH. The spectral analysis of photoplethysmography to evaluate an independent cardiovascular risk factor. Int J Gen Med. 2014;7:539-547. Published 2014 Dec 9. doi:10.2147/IJGM.S70892

42. Gandhi PG, Gundu HR. Detection of neuropathy using a sudomotor test in type 2 diabetes [published correction appears in Degener Neurol Neuromuscul Dis. 2015 Jul 16;5:73]. Degener Neurol Neuromuscul Dis. 2015;5:1-7. Published 2015 Jan 9. doi:10.2147/DNND.S75857

43. Maarek AA, Gandhi PG, Rao GHR. Identifying Autonomic Neuropathy and Endothelial Dysfunction in Type 2 Diabetic Patients. EC Neuropathy.2015;2.2:63-78.

44. Duprez DA, Cohn JN. Arterial stiffness as a risk factor for coronary atherosclerosis. Curr Atheroscler Rep. 2007;9(2):139-144. doi:10.1007/s11883-007-0010-y

45. Kelly AS, Wetzsteon RJ, Kaiser DR, Steinberger J, Bank AJ, Dengel DR. Inflammation, insulin, and endothelial function in overweight children and adolescents: the role of exercise. J Pediatr. 2004;145(6):731-736. doi:10.1016/j. jpeds.2004.08.004

46. Zhang H, Jiang L, Yang YJ, et al. Aerobic exercise improves endothelial function and serum adropin levels in obese adolescents independent of body weight loss. Sci Rep. 2017;7(1):17717. Published 2017 Dec 18. doi:10.1038/ s41598-017-18086-3

47. Davidson MB. Continuous Glucose Monitoring in Patients With Type 1 Diabetes Taking Insulin Injections. JAMA. 2017;317(4):363-364. doi:10.1001/jama.2016.20327

48. Bhavnani SP, Parakh K, Atreja A, et al. 2017 Roadmap for Innovation-ACC Health Policy Statement on Healthcare Transformation in the Era of Digital Health, Big Data, and Precision Health: A Report of the American College of Cardiology Task Force on Health Policy Statements and Systems of Care. J Am Coll Cardiol. 2017;70(21):2696-2718. doi:10.1016/j.jacc.2017.10.018

49. Steinberg JS, Varma N, Cygankiewicz I, et al. 2017 ISHNEHRS expert consensus statement on ambulatory ECG and external cardiac monitoring/telemetry [published correction appears in Heart Rhythm. 2018 Mar 28] [published correction appears in Heart Rhythm. 2018 Aug;15(8):1276]. Heart Rhythm. 2017;14(7):e55-e96. doi:10.1016/j.hrthm.2017.03.038

50. Ip JE. Wearable Devices for Cardiac Rhythm Diagnosis and Management. JAMA. 2019;321(4):337-338. doi:10.1001/ jama.2018.20437

51. MalmefeldtE, Rao GHR. Changing concepts of healthcare: Physical activity, Fitness and Wellness. EC Endocrinol Metab Rex.2019;4:238-250.

52. Malmefeldt E, Rao GHR. Noninvasive diagnostic tools: Cardiometabolic risk assessment and prediction. J Clin Cardiol Diagn. 2019;2(1):1-10.

53. Murakami H, Kawakami R, Nakae S, et al. Accuracy of Wearable Devices for Estimating Total Energy Expenditure: Comparison With Metabolic Chamber and Doubly Labeled Water Method. JAMA Intern Med. 2016;176(5):702-703. doi:10.1001/jamainternmed.2016.0152

54. Cortez NG, Cohen IG, Kesselheim AS. FDA regulation of mobile health technologies. N Engl J Med. 2014;371(4):372379. doi:10.1056/NEJMhle1403384

55. Quer G, Gouda P, Galarnyk M, Topol EJ, Steinhubl SR. Inter- and intraindividual variability in daily resting heart rate and its associations with age, sex, sleep, BMI, and time of year: Retrospective, longitudinal cohort study of 92,457 adults. PLoS One. 2020;15(2):e0227709. Published 2020 Feb 5. doi:10.1371/journal.pone.0227709

56. Tuttor M, von Stengel S, Kohl M, et al. High Intensity Resistance Exercise Training vs High Intensity Training to Fight Cardiometabolic Risk Factors in Overweight Men 30 50 Years Old. Frontiers in Sports and Active Living. June 16, 2020. doi: 10.3389/fspor.2020.0068.

57. Steinhubl SR. The future of individualized health maintenance. Nat Med. 2019;25(5):712-714. doi:10.1038/ s41591-019-0443-1

58. Palatini P, Casiglia E, Julius S, Pessina AC. High heart rate: a risk factor for cardiovascular death in elderly men. Arch Intern Med. 1999;159(6):585-592. doi:10.1001/ archinte.159.6.585

59. zmailova ES, Wagner JA, Perakslis ED. Wearable Devices in Clinical Trials: Hype and Hypothesis. Clin Pharmacol Ther. 2018;104(1):42-52. doi:10.1002/cpt.966

60. WHO CVD Risk Chart Working Group. World Health Organization cardiovascular disease risk charts: revised models to estimate risk in 21 global regions. Lancet Glob Health. 2019;7(10):e1332-e1345. doi:10.1016/S2214-109X(19)30318-3 61. Ueda P, Woodward M, Lu Y, et al. Laboratory-based and office-based risk scores and charts to predict 10-year risk of cardiovascular disease in 182 countries: a pooled analysis of prospective cohorts and health surveys. Lancet Diabetes Endocrinol. 2017;5(3):196-213. doi:10.1016/S22138587(17)30015-3

62. Pennells L, Kaptoge S, Wood A, et al. Equalization of four cardiovascular risk algorithms after systematic recalibration: individual-participant meta-analysis of 86 prospective studies. Eur Heart J. 2019;40(7):621-631. doi:10.1093/ eurheartj/ehy653

63. Rowland SP, Fitzgerald JE, Holme T, Powell J, McGregor A. What is the clinical value of mHealth for patients? NPJ Digit Med. 2020;3:4. Published 2020 Jan 13. doi:10.1038/ s41746-019-0206-X 